\title{
Remerciements à nos évaluateurs de 2015
}

Nous tenons à remercier les personnes suivantes pour leur contribution inestimable en tant qu'évaluateurs pour la revue Promotion de la santé et prévention des maladies chroniques au Canada en 2015. Leur expertise contribue grandement à la qualité de notre revue et à la diffusion des nouvelles connaissances auprès de la communauté scientifique, au Canada comme à l'échelle internationale.

José Almirall

Shazhan Amed

Tracie Barnett

Lana Beasley

Julie Bernier

Beatrice Boucher

Aimée Campeau

Yue Chen

Kristin Connor

Sarah Connor Gorber

Chris de Gara

Jessica Di Giovanni

Maureen Dobbins

Frank Elgar

Tara Flemington

Sarah Flicker

Robert Gates

Danijela Gasevic

Jenny Gray

Martine Hébert

Kathleen Hegadoren

Margaret Herbert

Shelly L. Jackson

Ian Janssen

Teresa Janz

Janet Elizabeth Jull
Lisa Kakinami
Glenn Keays
Colin King
Richard Krugman
Eric Lavigne
Allana LeBlanc
Isra Levy
Karen Liller
Larisa Lotoski
Wei Luo
Patti-Jean Naylor
Candace Nykiforuk
Alison Macpherson
Steven McFaull
Nazeem Muhajarine
Gillian Mulvale
Martin Noël
Lawrence Paszat
Scott B. Patten
Louise Pelletier
Cheryl Peters
Jennifer Petkovic
William Pickett
Robert Platt

Keith Power

Robert Prosser

Kirstein Rummery

Kelly Russell

Tara Sampalli

Travis Saunders

Jerry A. Schultz

Kevin Schwartz

Mark Smith

Richard Stanwick

Michael Stevenson

Larry Svenson

Wendy Thompson

Ellen Toth

Paul Veugelers

Charlotte Waddell

Christine Walsh

Darren Warburton

Sharon Warren

Christine White

Helene White

Andreas T. Wielgosz

Russell Wilkins 